\title{
Performance analysis of planar microcoils for biomedical wireless power transfer links
}

\author{
S KRISHNAPRIYA ${ }^{1, * 0}$, HIMANSHU CHANDRAKAR ${ }^{1}$, RAMA S KOMARAGIRI $^{2}$ and \\ K J SUJA ${ }^{1}$ \\ ${ }^{1}$ Department of Electronics and Communication Engineering, National Institute of Technology, Calicut, India \\ ${ }^{2}$ Department of Electronics and Communication Engineering, Bennett University, Noida, India \\ e-mail: s.kpriya2007@gmail.com
}

MS received 14 October 2018; revised 6 May 2019; accepted 6 June 2019

\begin{abstract}
Planar microcoils are significant components of inductive devices used for biomedical wireless power transfer applications. Biomedical devices implanted inside the human body needs to be powered up at regular intervals which can be done electromagnetically through a planar microcoils' setup. Different types of planar microcoils such as spiral and non-spiral planar microcoils are compared in this work in terms of their electrical parameters. An analytical model is also developed which is validated using experimental results. The fabrication advantages and low power dissipation of non-spiral coil structures make them a strong alternative for conventional spiral planar coils. Though the magnitude of magnetic flux density is slightly lesser for non-spiral coils, series resistance required for wireless power link is found to be better for the same. The fabrication of nonspiral planar microcoils of various geometries is shown using a single mask level. Coupling factors of various wireless power links are also simulated using different non-spiral planar microcoil geometries to select the optimum geometry for the wireless power link application.
\end{abstract}

Keywords. Non-spiral planar microcoil; magnetic flux density; wireless power link; coupling factor.

\section{Introduction}

Wireless power transfer schemes are acquiring more significance for biomedical implanted devices inside the human body as it can avoid transcutaneous wiring and replacement of device batteries. Planar microcoils are the primary components of an inductive wireless power link [1]. Reduction of the device volume is made possible using planar microcoils [2-4]. The efficiency of a wireless link depends on geometrical parameters of the microcoils, the distance between primary and secondary coils, the coupling between the coils and self and mutual inductance of the coils. Optimized coil design and knowledge of electrical characteristics of the planar microcoils are required to implement wireless power transfer devices for biomedical applications [1,5].

Planar microcoils can be classified into spiral and nonspiral types where a spiral type requires at least two metal levels for interconnections as shown in figure 1 [4]. The two contact leads are coplanar for a non-spiral planar coil, non-spiral microcoil fabrication requires only a single mask process as shown in figure 2 thus reducing process complexity. Moreover, non-spiral coils are advantageous with

*For correspondence

Published online: 26 July 2019 regards to its power consumption, as the resistance of nonspiral coils is smaller than that of its spiral counterpart [6-11]. As the non-spiral planar microcoil structure is similar to a set of short-circuited parallel conductors, the coil resistance is very low which leads to reduced Joule heating losses $[9,10]$.

This paper reports the analysis of spiral and non-spiral planar microcoils of five different geometries i.e. circular, square, tapered square, hexagonal, and octagonal geometries. To the best of our knowledge, comparative studies of spiral and non-spiral planar microcoils of different geometries are previously unpublished. Through this work, a total of ten planar microcoil types are compared in terms of their electrical parameters. Finite element analysis of various coil types is performed using COMSOL Multiphysics ${ }^{\circledR}$ software using which magnetic field distributions and electrical parameters of the various coil geometries are analysed. The essential steps for fabrication of non-spiral planar microcoils are discussed. Microfabrication of the five non-spiral coil geometries is performed in a single metal level. Electrical characterisation results of the fabricated coils are used to validate the analytical model. Also, a wireless power transfer link is realized using various non-spiral planar coil types to select the optimum geometry for obtaining higher coupling factors of the link. 


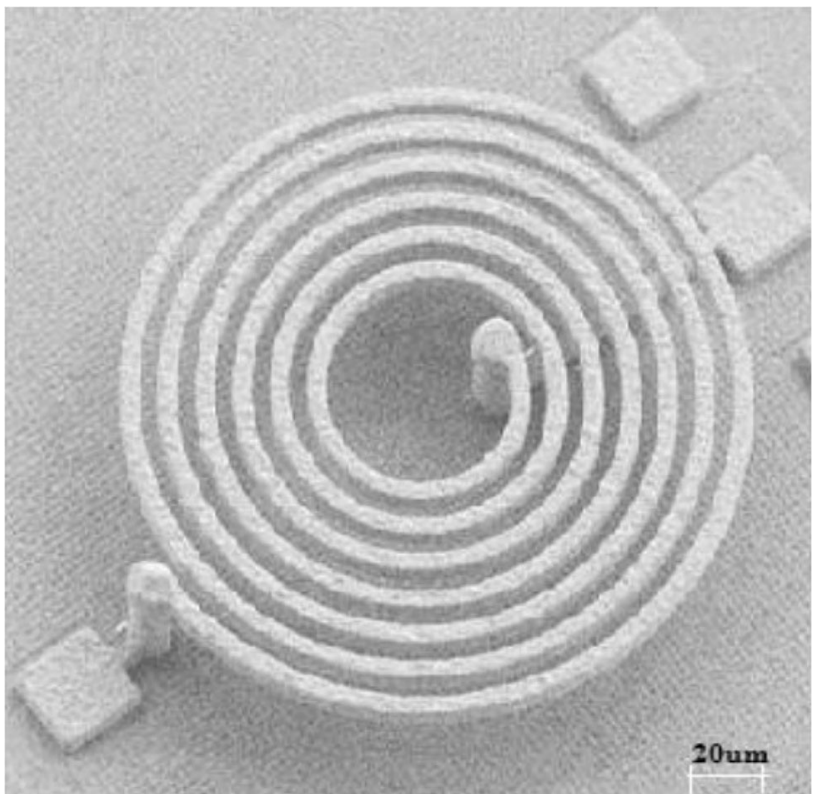

Figure 1. Micrograph of spiral planar microcoil of circular geometry.

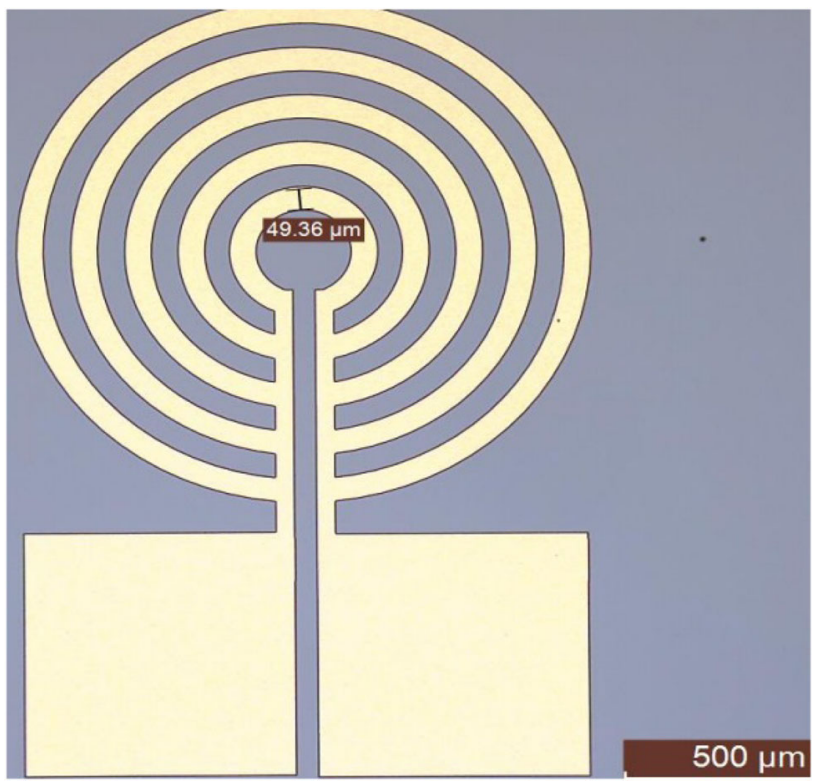

Figure 2. Micrograph of non-spiral planar microcoil of circular geometry.

\section{Electrical parameters of non-spiral planar microcoils}

Major electrical parameters of a planar microcoil include coil inductance, series resistance of the coil and magnetic field the coil can generate. Series resistance of the coil $\left(R_{s}\right)$ determines its Joule power dissipation. Magnetic field generated or detected by the coil depends upon the coil inductance $(L)$. Various geometrical parameters of the coil such as diameter of the innermost turn $\left(D_{1}\right)$, diameter of the outermost turn $(D)$, pitch $(p)$, coil width $(b)$ and height (h) are shown in figure 3.

Series Resistance can be divided into the two parts as one depending on frequency and another independent of frequency. The geometrical parameters of the coil mainly determine the series resistance. Generally, the shape of wire used in Micro-electro-mechanical systems (MEMS) is not round but rectangular in shape with a width $b$ and height $h$ $[1,2]$. The frequency independent coil resistance can be expressed as shown in Eq. (1) where $l$ is the length of the coil and $\rho$ is the resistivity of the coil material. Equation (2) shows the frequency dependent series resistance which is significant at higher frequencies of coil operation when the skin effect becomes dominant [1]. $\alpha$ is the fill ratio which is the ratio of $D$ to $D_{1}$.

$$
R_{s}=\frac{\rho l}{h b}
$$

$$
R_{s}=\rho D^{2} \frac{1-\alpha^{2}}{p}\left[\frac{1}{h b}\right]+\sqrt{\frac{\omega \mu}{8 \rho}}\left[\frac{1}{h+b}\right]
$$

Analytic formula for self inductance of square coil $\left(L_{s}\right)$ with single turn is given by Eq. (3) [1].

$$
L_{s}=\frac{2 \mu D}{\pi}\left[\ln \frac{4 D}{b+h}+0.894 \frac{b+h}{4 D}-0.660\right]
$$

Self inductances of other coil geometries such as circular, hexagonal and octagonal can be determined by the ratio of area with respect to the square loop as shown in Eqs. (4)(6) [12]. Therefore, self inductance of circular coil $\left(L_{c}\right)$ (in Hentry) is calculated with respect to Eq. (3) which is given by Eq. (4).

$$
L_{c}=\frac{\mu D}{2}\left[\ln \frac{4 D}{b+h}+0.894 \frac{b+h}{4 D}-0.660\right]
$$

Similarly, self inductance of hexagonal coil $\left(L_{h}\right)$ is found out which is given by Eq. (5) and that of octagonal coil $\left(L_{o}\right)$ is given by Eq. (6).

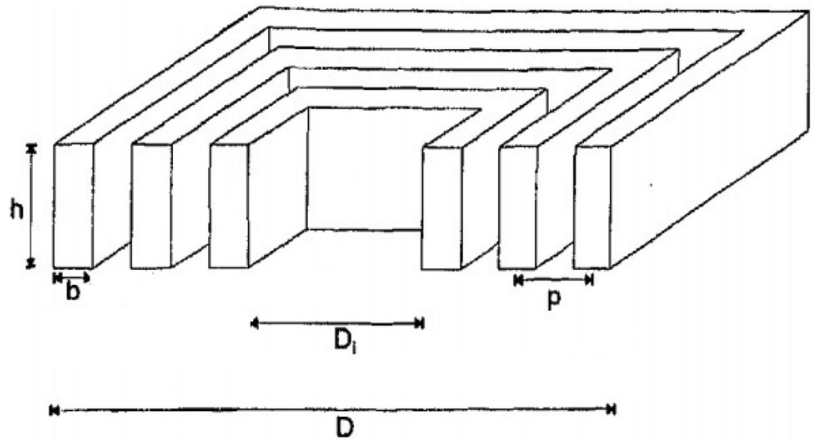

Figure 3. Schematic of a planar microcoil of square geometry showing different physical parameters. 


$$
\begin{array}{r}
L_{h}=\frac{1.3 \mu D}{8 \pi}\left[\ln \frac{4 D}{b+h}+0.894 \frac{b+h}{4 D}-0.660\right] \\
L_{o}=\frac{1.415 \mu D}{\pi}\left[\ln \frac{4 D}{b+h}+0.894 \frac{b+h}{4 D}-0.660\right]
\end{array}
$$

By inspecting Eqs. (3)-(6), coil inductance can be arranged in the descending order of their magnitudes as in the following order: square, circular, octagonal and hexagonal.

Series coil resistance directly depends upon the length of a microcoil which in turn influences the DC power dissipation of the coil. Length of various coil geometries are calculated using Eqs. (7)-(10). Figure 4 shows schematic diagrams of square and hexagonal coils which is helpful to find out the approximate expressions for coil lengths. Geometrical parameters considered for calculation are shown in table 1 with number of turns, $n$ is fixed as five. The length of a square coil is given by Eq. (7).

$$
l=\sum_{i=1}^{n} 7 r+8(i-1) p
$$

where $r$ is radius of the innermost turn (i.e., $\mathrm{r}=D_{1} / 2$ ) and $p$ is the pitch of the microcoil. The length of a hexagonal coil is given by Eq. (8).

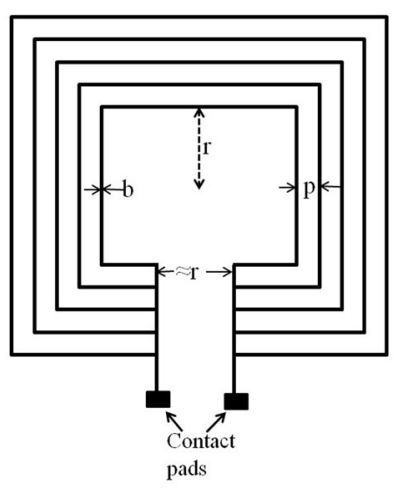

(a)

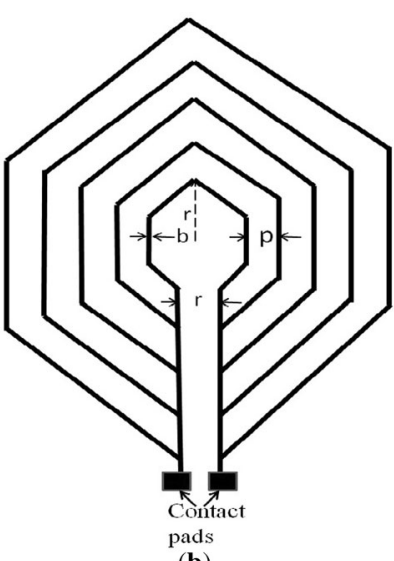

(b)
Figure 4. Schematic of planar microcoils for length calculation of (a) square geometry, (b) hexagonal geometry.

Table 1. Geometrical parameters of the various coil geometries under comparative study.

\begin{tabular}{lc}
\hline Geometrical perimeter & Value in $\mu \mathrm{m}$ \\
\hline Width & 100 \\
Pitch & 100 \\
Height & 10 \\
Internal diameter & 200 \\
External diameter & 1600 \\
\hline
\end{tabular}

$$
l=\sum_{i=1}^{n} 5 r+6(i-1) p
$$

The length of a octagonal and circular coil is given by Eq. (9) and Eq. (10) respectively.

$$
\begin{gathered}
l=\sum_{i=1}^{n} 5.13 r+8(i-1) p \\
l=\sum_{i=1}^{n} 5.28 r+6.28(i-1) p
\end{gathered}
$$

Based on the length expressions, it is observed that the length of square coil is the highest followed by hexagonal coil, octagonal coil and the circular coil for the same set of geometrical parameters as shown in table 1 . As the circular coil possesses the lowest length, circular coil has the lowest series coil resistance and the lowest DC power dissipation among the different coil geometries under consideration.

\section{Finite element analysis of planar microcoils}

Magnetic field distributions in planar microcoils of spiral and non-spiral types of square, tapered square, circular, hexagonal and octagonal geometries are studied using finite element analysis (FEA) in Comsol Multiphysics ${ }^{\circledR}$. Geometrical parameters of the coil geometries under study are taken as uniform and shown in table 1 . Number of turns is fixed to be five. Magnitude of excitation current is set as 0.5 A.

Simulations are performed at a low frequency of $10 \mathrm{kHz}$ considering biomedical compatibility [13, 14].

Planar spiral microcoils of various geometries such as square, hexagonal, octagonal and circular are simulated to obtain the magnetic field distribution which are shown in figure 5. The input excitation current flows uniformly through all of the coil turns in a spiral planar microcoil resulting in a uniform field distribution across the turns as shown in figure 5 whereas a non-spiral planar coil field distribution is non-uniform as shown in figure 7.

The reason behind the non-uniform field distribution in non-spiral planar microcoils is the asymmetric current division among the coil turns as the turns are interconnected as a set of parallel short-circuited conductors. The innermost turn in a non-spiral planar microcoil possesses the least resistance due to which majority of the input current is flowing through the innermost turn. This causes the magnetic field to be maximum at the innermost turn as observed from the FEA results shown in figure 7. The resistance of the coil turns increases gradually towards the outer turns which in turn causes the magnetic field to decrease towards the outer turns as shown in figure 7.

A tapered square geometry is also experimented in this work and the magnetic field distributions in the tapered 


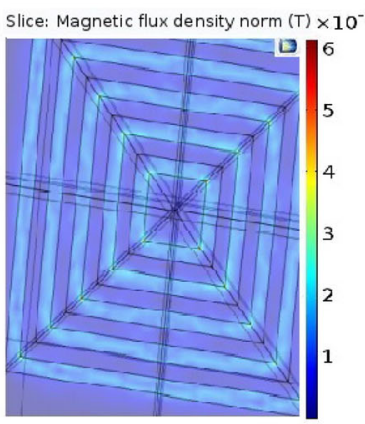

(a)

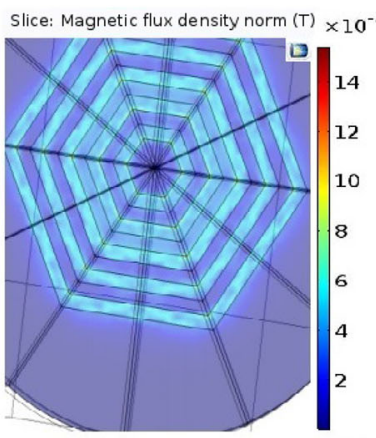

(b)

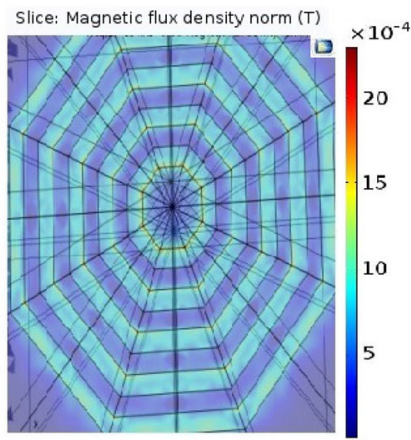

(c)

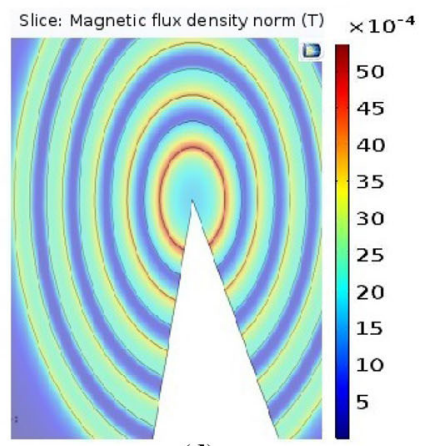

(d)

Figure 5. Magnetic field distribution of planar spiral microcoils of various geometries. (a) Square. (b) Hexagonal. (c) Octagonal. (d) Circular.

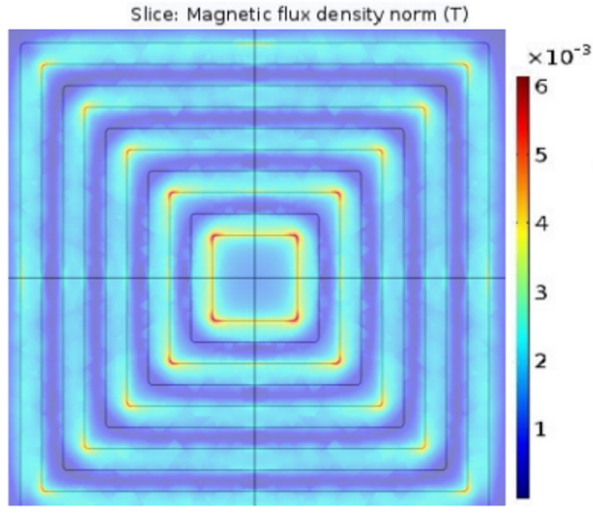

Figure 6. Magnetic field distribution of planar tapered square spiral microcoil.

square spiral and non-spiral planar microcoil geometries are shown in figures 6 and 8 . The tapered geometry provides an improvement in the peak magnetic field compared to the other coil geometries such as square, hexagonal and octagonal coils. As the tapered square coil structure doesn't have sharp bends or corners, magnetic field distribution is more uniform than that in non-tapered structures. As circular geometry does not have sharp edges or corners as in the case of other geometries under consideration, the simulated magnetic field distribution is found to be uniform along a coil turn in circular coils as shown in figures 5(d) and 7(d). In non-circular geometries, the field is shown to be denser at every inner corner of the coil turns. Also, the coil resistance is the smallest in the case of non-spiral circular coil geometry. Therefore, optimal magnetic field with minimal coil resistance is offered by circular non-spiral microcoil as observed from the comparative studies (figure 8).

Table 2 shows comparison between various planar coil geometries in terms of their peak magnetic flux density (B), inductance $(\mathrm{L})$ and ac series resistance $(\mathrm{R})$ at a frequency of $10 \mathrm{kHz}$ of input excitation. Tapered square spiral microcoil is found to generate the highest peak magnetic field among the various coil types considered. However, the series resistance observed for the same is large. Non-spiral planar coils are found to show lower resistance compared to their spiral counterpart.

As circular geometry does not have sharp edges or corners as in the case of other geometries under consideration, the simulated magnetic field distribution is found to be uniform along a coil turn in circular coils as shown in figures 5(d) and 7(d). In non-circular geometries the field is shown to be denser at every inner corner of the coil turns. Also, the coil resistance is the smallest in the case of nonspiral circular coil geometry. Therefore, optimal magnetic

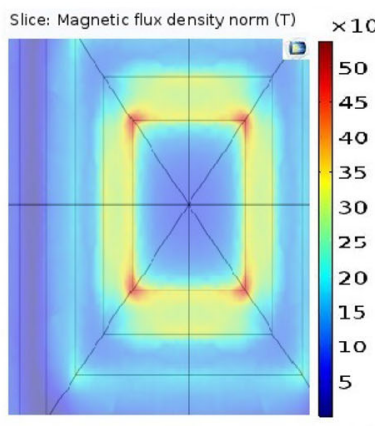

(a)

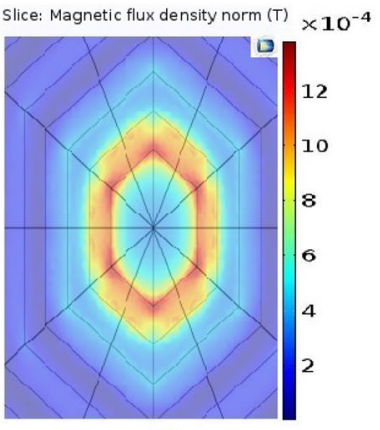

(b)

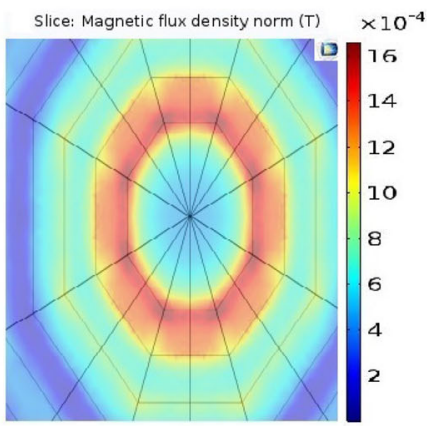

(c)

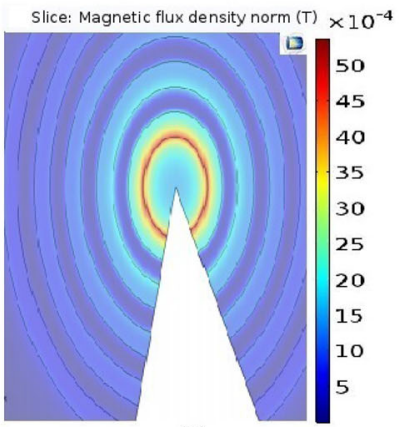

(d)

Figure 7. Magnetic field distribution of planar non-spiral microcoils of various geometries. (a) Square. (b) Hexagonal. (c) Octagonal. (d) Circular. 


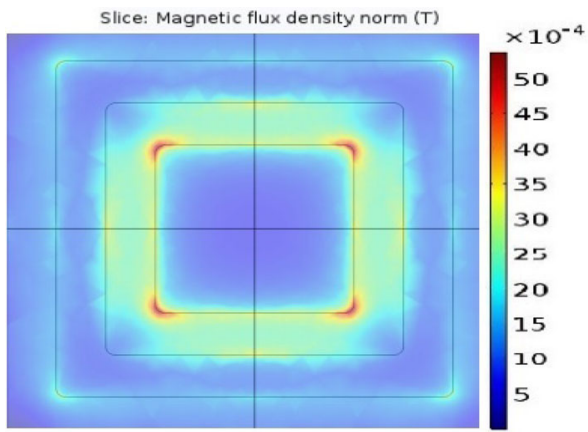

Figure 8. Magnetic field distribution of planar tapered square non-spiral microcoil.

Table 2. Comparison of electrical parameters of various planar microcoil geometries.

\begin{tabular}{lcccc}
\hline Geometry & Coil type & B $(\mathrm{mT})$ & $\mathrm{L}(\mathrm{mH})$ & $\mathrm{R}(\mathrm{ac})[\Omega]$ \\
\hline Square & Spiral & 6.05 & 0.83 & 2.05 \\
& Non-spiral & 5.24 & 0.59 & 0.25 \\
Hexagonal & Spiral & 1.27 & 0.44 & 1.925 \\
& Non-spiral & 1.2 & 0.36 & 0.21 \\
Octagonal & Spiral & 2.01 & 0.51 & 1.84 \\
& Non-spiral & 1.54 & 0.3 & 0.20 \\
Circular & Spiral & 5.8 & 0.53 & 1.37 \\
& Non-spiral & 4.9 & 0.47 & 0.172 \\
Tapered square & Spiral & 7.21 & 0.88 & 1.787 \\
& Non-spiral & 6.71 & 0.6 & 0.196 \\
\hline
\end{tabular}

Table 3. Highlights of the non-spiral planar microcoil geometries.

\begin{tabular}{lc}
\hline Geometry & Properties \\
\hline $\begin{array}{l}\text { Square } \\
\text { Hexagonal } \\
\text { Octagonal } \\
\begin{array}{l}\text { Circular } \\
\text { The lowest magnetic field with high resistance } \\
\text { Lapered } \\
\text { square }\end{array} \\
\text { Optimal magnetic field with the lowest } \\
\text { resistance }\end{array}$ \\
\hline
\end{tabular}

field with minimal coil resistance is offered by circular nonspiral microcoil as observed from the comparison studies. Table 3 highlights the major features of the various nonspiral planar coil geometries.

\section{Microfabrication of non-spiral planar microcoils}

A simplified fabrication process is experimented in this work for manufacturing non-spiral planar microcoils. Microfabrication of non-spiral planar microcoils is performed using a single mask and metal level process. A four inch, p-type silicon wafer is chosen as the substrate on

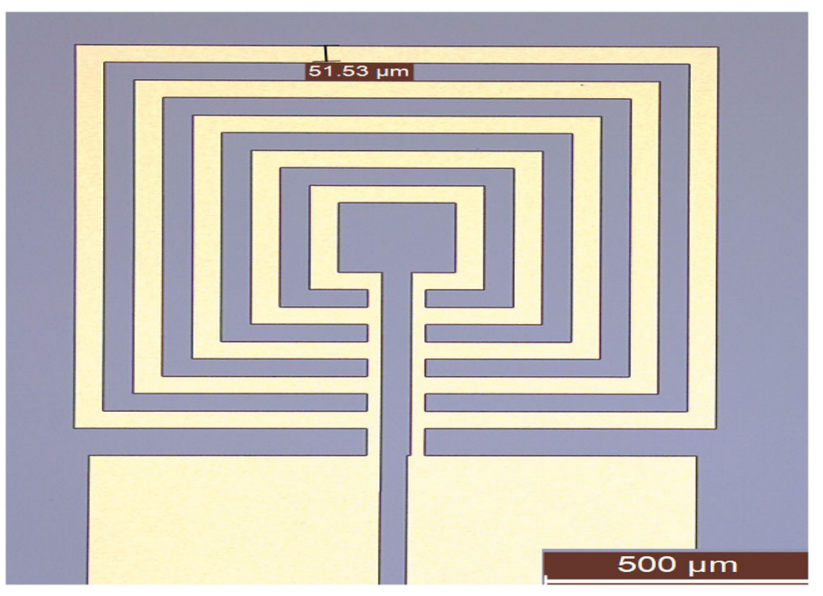

Figure 9. Micrograph of non-spiral planar square microcoil.

which microcoils are fabricated. Wafer was undergone oxidization with $1 \mu \mathrm{m}$ thick thermal oxide in an oxidation furnace. Aluminium is chosen as the coil material and Aluminium metallization of $1 \mu \mathrm{m}$ thickness was deposited on the oxide by electron beam evaporation. Coil patterns were then transferred to the photoresist coated on the Aluminium layer, and then developed. The final steps include wet etching of unwanted portions of Aluminium and stripping of photoresist. Coil geometries of circular, square, tapered square, hexagonal and octagonal shapes are fabricated on a common oxidized Silicon substrate. Micrographs of the non-spiral planar microcoils of various geometries are shown in figures 9 and 10.

\section{Experimental validation of the electrical model}

Electrical characterization of the fabricated non-spiral planar microcoils was performed using Agilent Semiconductor Device Analyzer (SDA). Coil inductance and series resistance were measured at a low frequency of $10 \mathrm{kHz}$ considering the wireless power transfer scenario for biomedical devices. The measured values are compared with the electrical model presented in section 2 to validate the model. The results are shown in figures 11 and 12. The disparity is found to be less than a percentage value of 10 .

\section{Finite element analysis of wireless power transfer link using non-spiral planar microcoils}

Considering the low coil resistance and fabrication advantages of non-spiral coils, non-spiral coils are selected to implement a wireless power link to medical implants. A wireless power transfer link is realized which can be used for biomedical devices to power up medical implanted equipment inside human body. 


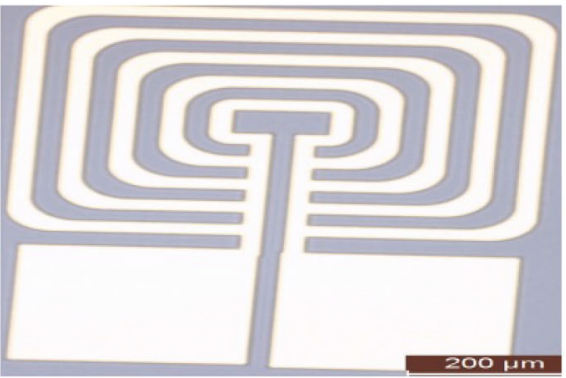

(a)

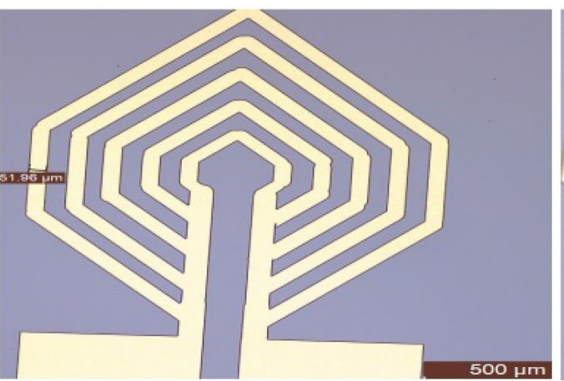

(b)

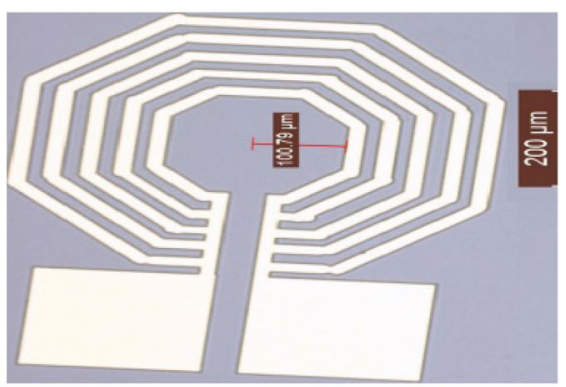

(c)

Figure 10. Micrographs of non-spiral microcoils of various geometries. (a) Tapered square. (b) Hexagonal. (c) Octagonal.

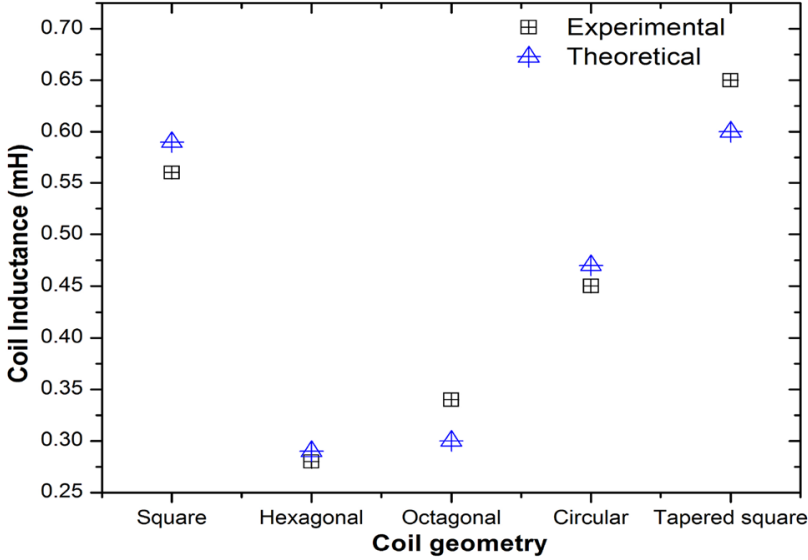

Figure 11. Comparison of coil inductance between experimental results and electrical model.

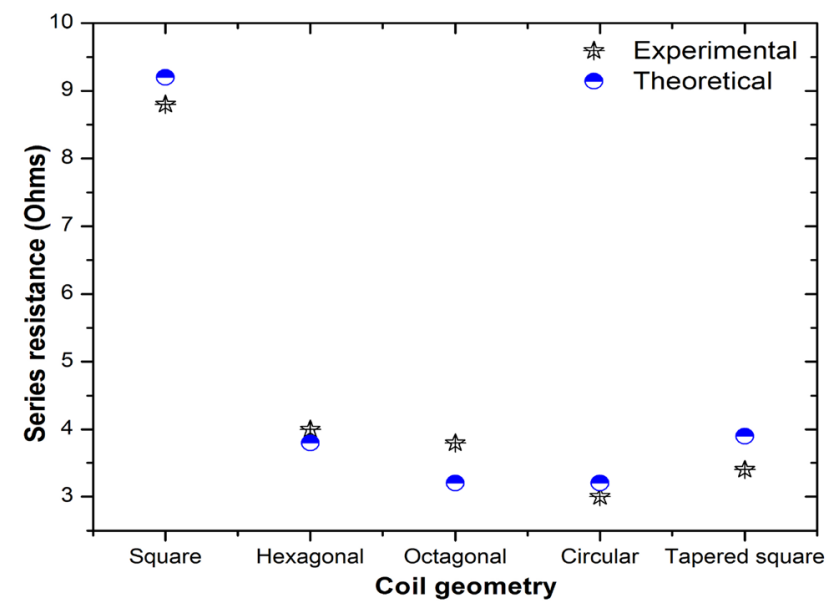

Figure 12. Comparison of series resistance between experimental results and electrical model.

Two sets of coils are considered one as primary coil which when energized will couple power to the secondary coil kept within a millimeter distance. Considering the

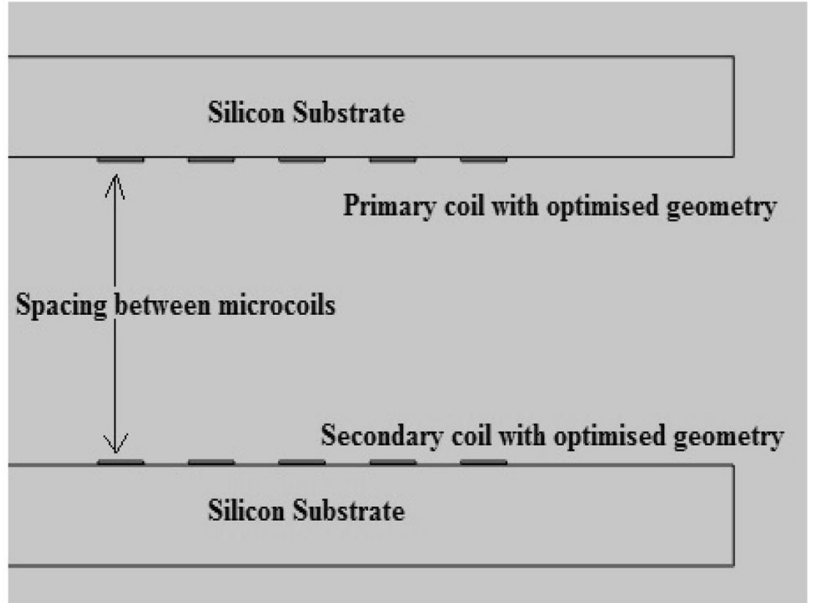

Figure 13. Cross section of the proposed wireless power transfer system using non-spiral planar microcoils.

human epidermis thickness ranging from $50 \mu \mathrm{m}$ on the eyelid to a few $\mathrm{mm}$ on the back, the spacing between primary and secondary coils is varied from $50 \mu \mathrm{m}$ to $1 \mathrm{~mm}$ $[15,16]$.

The coils were enclosed in air domain considering the magnetic permeability of human tissue which is same as that of air, as the human body is not perturbed by external magnetic fields at low frequencies. The primary coil is excited with an alternating current of amplitude $100 \mathrm{~mA}$ at a frequency of $10 \mathrm{kHz}$ considering biomedical compatibility and safety requirements required for biomedical applications $[14,16]$. Current in the secondary receiving coil is set as zero.

Primary and secondary coils of height $1 \mu \mathrm{m}$, width $50 \mu \mathrm{m}$ and pitch $50 \mu \mathrm{m}$ are placed vertically one above each other as shown in figure 13. Voltage induced in the secondary coil $\left(V_{2}\right)$ is simulated by applying a primary coil input current.

The mutual inductance between two coils is calculated between two coils using Eq. 11. 


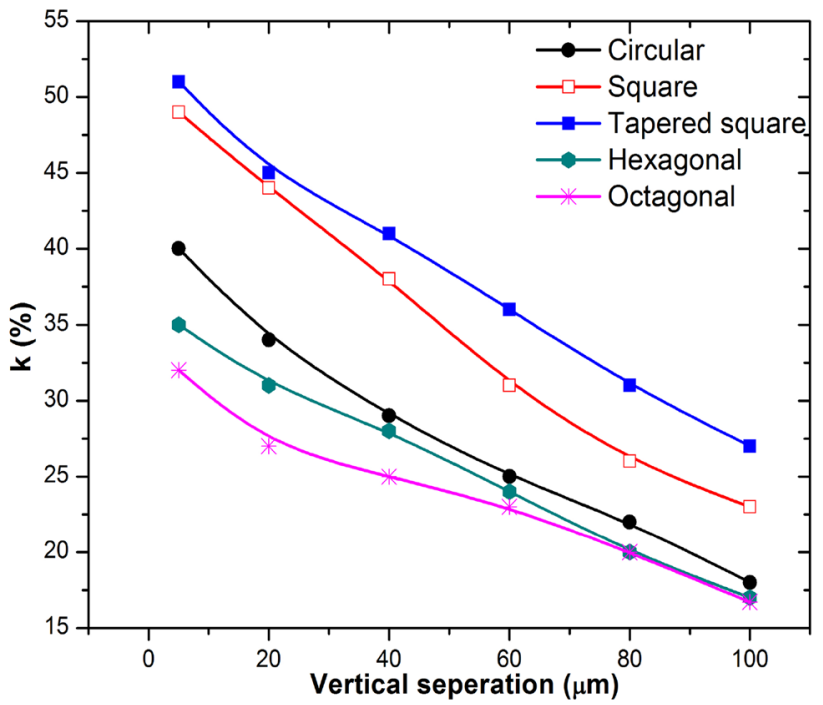

Figure 14. Coupling factor as a function of separation between primary and secondary coils for different coil geometries.

$$
M=V_{2} / \omega I_{1}
$$

where $V_{2}$ is secondary voltage and $I_{1}$ is the primary coil current. The coupling factor between two coils can be calculated by using Eqs. 11 and 12 .

$$
k=M / \sqrt{L_{1} L_{2}}
$$

Where $\mathrm{M}$ is mutual inductance between two coils and $L_{1}$ and $L_{2}$ are the self inductances of primary and secondary coils.

Coupling factors calculated as a function of the spacing between the primary and secondary coils is shown in figure 14 for various non-spiral planar coil geometries. The tapered square non-spiral coil possesses the highest values of coupling factors with octagonal coil values being the lowest. Percentage $\mathrm{k}$ values as high as 55 is obtained using tapered square geometry. The ways to further improve the power coupling factor are currently under study.

\section{Conclusion}

Planar microcoils of spiral and non-spiral types of various geometries are analysed in this work with regard to their electrical parameters. Comparison studies shows that the circular non-spiral coil yields optimum magnetic field with minimum series coil resistance required for biomedical wireless power links. Microfabrication process of the nonspiral planar coils is presented using a single metal level of interconnections to show the fabrication easiness of the non-spiral planar coil types. The proposed analytical model for the coil geometries is found to be compatible with the experimental results. A wireless power transfer link is also set up and analysed using different non-spiral planar coil geometries in order to compare the coupling factors under various coil geometries. Tapered square microcoil shows the highest coupling factors among the various non-spiral coil geometries compared. Circular non-spiral coils can be considered as suitable candidates for the wireless power links as the circular coils possess coupling factors closer to that of tapered square microcoils with a uniform field distribution and the lowest series coil resistance.

\section{Acknowledgements}

This research was performed using facilities at CeNSE, Indian Institute of Science, Bengaluru, funded by the Ministry of Electronics and Information Technology (MeitY), Govt. of India.

\section{References}

[1] Amato M, Dalena F, Coviello C, De Vittorio M and Petroni S 2013 Modeling, fabrication and characterization of microcoils as magnetic inductors for wireless power transfer. $M i$ croelecron. Eng. 111:143-148

[2] Eroglu S, Friedman G and Magin R L 2001 Estimate of losses and signal- to-noise ratio in planar inductive microcoil detectors used for NMR. IEEE Transactions on Magnetics. 37(4): 2787-2789

[3] Krishnapriya S, Komaragiri R and Suja K J 2016 Significance of non-spiral planar microcoils over spiral coils for biomedical applications. In: Proceedings of 2016 IEEE International Conference on Emerging Electronics, pp. 1-4

[4] Pan M, He Y, Xie R and Zhou 2014 The influence of MEMS on electromagnetic NDT. In: Non-destructive Evaluation/ Testing, IEEE Far East Forum, pp. 363-367

[5] Uttamchandani D 2013 Handbook of MEMS for Wireless and Mobile Applications, Elsevier

[6] Krishnapriya S, Komaragiri R and Suja K J 2017 Non-spiral planar microcoils for biomedical applications. In: IEEE 12th International Conference on Nano/Micro Engineered and Molecular Systems (NEMS), pp. 780-783

[7] Krishnapriya S, Komaragiri R and Suja K J 2018 Electromagnetic microactuators using non-spiral planar microcoils for robotic applications. Procedia Comput. Sci. 133: 545-552

[8] Wu W P, Lu R S, Zhou X L and Ni Z H 2013 The nuclear magnetic resonance probe based on a printed circuit board planar microcoil. In: Proceedings of 2013 IEEE International Conference on Applied Superconductivity and Electromagnetic Devices, pp. 169-172

[9] Pawinanto R E, Yunas J, Majlis B Y and Hamzah A A 2013 Finite element analysis on magnetic force generation of electromagnetic microactuator for micropump. In: 2013 IEEE Regional Symposium on Micro and Nanoelectronics (RSM), pp. 25-28

[10] Krishnapriya S, Komaragiri R S and Suja K J 2017 Modeling and simulation of non-spiral coil for magnetic sensing applications. J. Mech. Sci. Technol. 31(11): 5415-5421

[11] Krishnapriya S, Komaragiri R and Suja K J 2019 Fabrication, characterization, and modelling of a novel via-less single 
metal level magnetic microcoil sensor for biosensing applications. Sens. Actuators A: Phys. 290: 190-197

[12] Liao S, Li W, Lin S, Tang K, Liou C and Tsou C 2018 A study of the effect of different coplanar dual-coil geometries on the performance of mutual inductance. Sens. Actuators A Phys. 269: 99-110

[13] Strasnick E, Yang J, Tanner K, Olwal A and Follmer S 2017 Reconfigurable tactile elements for dynamic a ordances and mobile interaction. In: Proceedings of the 2017 CHI Conference on Human Factors in Computing Systems, pp. 5075-5086
[14] Siauve N, Scorretti R, Burais N, Nicolas L and Nicolas A 2003 Electromagnetic fields and human body: a new challenge for the electromagnetic field computation. COMPEL Int. J. Comput. Math. Electr. Electron. Eng. 22(3):457-469

[15] Leuerer T and Mokwa W 2004 Planar coils with magnetic layers for optimized energy transfer in telemetric systems. Sens. Actuators A Phys. 116(3): 410-416

[16] Peratta C and Peratta A 2010 Modelling the Human Body Exposure to Elf Electric Fields, WIT Press 